\title{
Book Review: Holographic Entanglement Entropy
}

\author{
Ashkbiz Danehkar* \\ Department of Astronomy, University of Michigan, Ann Arbor, MI, United States
}

Keywords: AdS/CFT correspondence, holographic principle, quantum entanglement, quantum gravity, book review

\section{A Book Review on \\ Holographic Entanglement Entropy}

Mukund Rangamani, Tadashi Takayanagi, Lecture Notes in Physics Vol. 931 (Berlin: Springer Verlag), 2017, 246 pages, ISBN: hardback 978-3-319-52571-6, ebook 978-3-319-52573-0, doi: $10.1007 / 978-3-319-52573-0$

The entanglement entropy is a quantity to measure the correlation between two entangled quantum many-body subsystems in quantum field theories (QFT). The divergence of the entanglement entropy of an entangled many-body subsystem is proportional to its area, which is analogous to the Bekenstein-Hawking black hole entropy formula [1-3], where the entropy of a black hole is proportional to its event horizon area. The holographic correspondence between $d+1$-dimensional Anti-de Sitter (AdS) spacetime and conformal field theory in $d$ dimensions $\left(\mathrm{AdS}_{d+1} / \mathrm{CFT}_{d}\right.$ correspondence) $[4,5]$ points to the geometric counterpart of the entanglement entropy, the socalled holographic entanglement entropy [6-8], which is the topic of the new book by Rangamani and Takayanagi [9].

This textbook is part of the book series Lecture Notes in Physics published by Springer Nature aimed at providing up-to-date research and teaching materials for postgraduate students and researchers. The book covers the holographic view of the entanglement entropy in the AdS/CFT correspondence. The book is divided into four parts: quantum entanglement, holography and entanglement, entanglement and quantum dynamics, and quantum gravity. The basic formalism of entanglement in quantum mechanics was briefly introduced in the first part, which is necessary for understanding the entanglement entropy in the holographic dictionary. The second part of the book focuses on the entanglement entropy in holographic field theories, followed by their applications and recent developments in the third part of the book. The final part of the book focuses on the holographic map between quantum entanglement and geometric concepts such as the Einstein-Rosen bridge (ER), which could be associated with the underlying quantum system of gravity.

The most distinctive feature of quantum mechanics is the presence of quantum entanglement in such a way that the quantum state of groups of entangled particles are not independent, even over an unprecedented distance. This phenomenon, which is not present in classical physics, was referred to as "spooky action at a distance" by Einstein in his letter to Born in 1947 [10], which was first speculated in the Einstein-Podolsky-Rosen (EPR) thought experiment in 1935 [11-13]. The von Neumann entropy of the reduced density matrix $[14,15]$, referred to as the entanglement entropy, is used as a quantitative measure of entanglement in quantum mechanics. There is another quantity, called the Rényi entropy [16], capturing the moments of the reduced density matrix, which provides a measure of quantum purity of the system. In the first part of the book, the authors briefly reviewed the von Neumann and Rényi entanglement entropies and discussed them in the 
simplest quantum many-body system, i.e., two-dimensional conformal field theory $\left(\mathrm{CFT}_{2}\right)$.

The $\mathrm{AdS}_{d+1} / \mathrm{CFT}_{d}$ correspondence, which was proposed by Maldacena in 1997 [4, 5], relates quantum gravity in $d+1$-dimensional AdS spacetime to CFT in $d$ dimensions. Accordingly, type IIB supergravity in $\mathrm{AdS}_{5}$ with 5 -D compact sphere is equivalent to $\mathcal{N}=4$ super Yang-Mills theory in 4 dimensions [17]. The AdS/CFT correspondence is one of the examples of the holographic principle, which states that the degrees of freedom in $d+1$-dimensional quantum gravity are comparable to those of $d$ - dimensional quantum manybody systems $[18,19]$. Thus, one would expect to geometrically imagine the gravitational picture of the QFT entanglement entropy according to the AdS/CFT correspondence. This idea was elaborated in the Ryu-Takayanagi (RT) proposal in 2006 $[6,7]$, which was generalized to time-dependent states in the Hubeny-Rangamani-Takayanagi (HRT) prescription in 2007 [8]. A comprehensive review on the formulations and proprieties of the RT/HRT proposals, referred to as the holographic entanglement entropy, is presented in the second part of the book.

In the view point of the AdS/CFT correspondence, a black hole in $\mathrm{AdS}_{d+1}$ is dual to a $\mathrm{CFT}_{d}$ at a finite temperature. Accordingly, a holographic counterpart of a quantum quench, which is an important class of non-equilibrium physics in QFT, corresponds to the black hole creation in AdS. Moreover, the eternal Schwarzschild-AdS $S_{d+1}$ black hole could be equivalent to the thermofield double state of the entanglement entropy in $\mathrm{CFT}_{d}$ [20]. These are some applications of the RT/HRT prescriptions, which were considered in the third part of the book.

The holographic principle implies that quantum entanglement is somehow associated with the building block of

\section{REFERENCES}

1. Bekenstein JD. Black holes and entropy. Phys Rev D. (1973) 7:2333-46. doi: 10.1103/PhysRevD.7.2333

2. Hawking SW. Particle creation by black holes. Commun Math Phys. (1975) 43:199-220. doi: 10.1007/BF02345020

3. Hawking SW. Particle creation by black holes. Commun Math Phys. (1976) 46:206. doi: 10.1007/BF01608497

4. Maldacena JM. The large $\mathrm{n}$ limit of superconformal field theories and supergravity. Adv Theor Math Phys. (1998) 2:231-52.

5. Maldacena J. The large-N limit of superconformal field theories and supergravity. Int $J$ Theor Phys. (1999) 38:1113-33. doi: 10.4310/ATMP.1998.v2.n2.a1

6. Ryu S, Takayanagi T. Holographic derivation of entanglement entropy from the anti de sitter space/conformal field theory correspondence. Phys Rev Lett. (2006) 96:181602. doi: 10.1103/PhysRevLett.96.181602

7. Ryu S, Takayanagi T. Aspects of holographic entanglement entropy. J High Energy Phys. (2006) 2006:045. doi: 10.1088/1126-6708/2006/08/045

8. Hubeny VE, Rangamani M, Takayanagi T. A covariant holographic entanglement entropy proposal. J High Energy Phys. (2007) 7:062. doi: 10.1088/1126-6708/2007/07/062

9. Rangamani M, Takayanagi T. Holographic Entanglement Entropy. Vol. 931 of Lecture Notes in Physics. Berlin: Springer Verlag; Springer International Publishing (2017).

10. Einstein A, Born M, Born H. The Born-Einstein Letters: Correspondence Between Albert Einstein and Max and Hedwig Born from 1916-1955, the gravitational spacetime. Accordingly, the ER bridge, which describes an internal wormhole connection between two distant, entangled black holes, is related to quantum entanglement prescribed by the EPR thought experiment, the so called "ER $=$ EPR" principle [21]. The connection between spacetime geometry and quantum entanglement was discussed in the final part of the book. The final chapter of the book summarized a class of tensor networks for ground states in a many-body system, called the multi-scale entanglement renormalization ansatz (MERA) [22] and the continuous MERA (cMERA) $[23,24]$. These tensor networks might help us develop a better understanding of the quantum nature of gravity.

The RT/HRT proposals prescribed by Hubeny, Rangamani, Ryu, and Takayanagi [6-8] over a decade ago are one of outcomes of the gauge/gravity or AdS/CFT correspondence proposed by Maldacena nearly two decades ago $[4,5]$, which generalizes the QFT entanglement entropy to the geometric configuration in gravitational field theories, and could be the base for the ER = EPR principle recently proposed by Susskind and Maldacena in 2013 [21]. The authors of this book, among those who introduced the RT/HRT prescriptions [6-8], have provided a comprehensive guide to the recent developments in the holographic entanglement entropy and its applications to gravitational theories. This book is therefore an excellent resource for getting acquainted with the relatively new topic of the holographic dual of the entanglement entropy under the gauge/gravity duality.

\section{AUTHOR CONTRIBUTIONS}

The author confirms being the sole contributor of this work and has approved it for publication.
With Commentaries by Max Born. Transl. by I. Born. London: Macmillan (1971).

11. Einstein A, Podolsky B, Rosen N. Can quantum-mechanical description of physical reality be considered complete? Phys Rev. (1935) 47:777-80. doi: 10.1103/PhysRev.47.777

12. Schrödinger E. Discussion of probability relations between separated systems. Proc Cambridge Philos Soc. (1935) 31:555. doi: 10.1017/S0305004100013554

13. Schrödinger E. Probability relations between separated systems. Proc Cambridge Philos Soc. (1936) 32:446. doi: 10.1017/S0305004100019137

14. von Neumann J. Mathematische Grundlagen der Quantenmechanik. Berlin: Springer (1932).

15. von Neumann J. Mathematical Foundations of Quantum Mechanics. Princeton, NJ: Princeton University Press (1955).

16. Rényi A. On measures of entropy and information. In: Proceedings of the Fourth Berkeley Symposium on Mathematical Statistics and Probability, Volume 1: Contributions to the Theory of Statistics. Berkeley, CA: University of California Press (1961). p. 547-61.

17. Witten E. Anti-de Sitter space and holography. Adv Theor Math Phys. (1998) 2:253-91.

18. 't Hooft G. Dimensional reduction in quantum gravity. Conf Proc. (1993) C930308:284-96.

19. Susskind L. The world as a hologram. J Math Phys. (1995) 36:6377-96. doi: $10.1063 / 1.531249$

20. Hartman T, Maldacena J. Time evolution of entanglement entropy from black hole interiors. J High Energy Phys. (2013) 2013:14. doi: 10.1007/JHEP05(2013)014 
21. Maldacena J, Susskind L. Cool horizons for entangled black holes. Fortsch Phys. (2013) 61:781-811. doi: 10.1002/prop. 201300020

22. Vidal G. Entanglement renormalization. Phys Rev Lett. (2007) 99:220405. doi: 10.1103/PhysRevLett.99.220405

23. Nozaki M, Ryu S, Takayanagi T. Holographic geometry of entanglement renormalization in quantum field theories. J High Energy Phys. (2012) 2012:193. doi: 10.1007/JHEP10(2012)193

24. Haegeman J, Osborne TJ, Verschelde H, Verstraete F. Entanglement renormalization for quantum fields in real space. Phys Rev Lett. (2013) 110:100402. doi: 10.1103/PhysRevLett.110.100402
Conflict of Interest Statement: The author declares that the research was conducted in the absence of any commercial or financial relationships that could be construed as a potential conflict of interest.

Copyright (C) 2019 Danehkar. This is an open-access article distributed under the terms of the Creative Commons Attribution License (CC BY). The use, distribution or reproduction in other forums is permitted, provided the original author(s) and the copyright owner(s) are credited and that the original publication in this journal is cited, in accordance with accepted academic practice. No use, distribution or reproduction is permitted which does not comply with these terms. 